Final Report for Period: 09/2008 - 08/2009

Principal Investigator: O'Neil, Thomas M.

Organization: U of Cal San Diego

Submitted By:

Driscoll, C. Fred - Co-Principal Investigator

Title:

FUNDAMENTAL PROCESSES IN PLASMAS

\section{Senior Personnel}

Name: O'Neil, Thomas

Worked for more than 160 Hours: Yes

Contribution to Project:

Name: Driscoll, C. Fred

Worked for more than 160 Hours: Yes

Contribution to Project:

Name: Dubin, Daniel H.E.

Worked for more than 160 Hours: Yes

Contribution to Project:

Post-doc

Name: Valentini, Francesco

Worked for more than 160 Hours: Yes

Contribution to Project:

Visiting postdoctoral scholar

Name: Bass, Eric

Worked for more than 160 Hours: Yes Contribution to Project:

Graduate Student

Name: Kuzmin, Stanislav

Worked for more than 160 Hours: Yes Contribution to Project:

Name: Shiga, Nobuyasu

Worked for more than 160 Hours: Yes Contribution to Project:

Name: Yu, Jonathan

Worked for more than 160 Hours: Yes Contribution to Project:

Name: Bass, Eric

Worked for more than 160 Hours: Yes
Submitted on: 11/30/2009

Award ID: 0354979

$\underline{\text { Project Participants }}$ 
Contribution to Project:

Name: Creveling, Daniel

Worked for more than 160 Hours: Yes

Contribution to Project:

Name: Quinn, John

Worked for more than 160 Hours: Yes

Contribution to Project:

Name: Anderson, Michael

Worked for more than 160 Hours: Yes

Contribution to Project:

Name: Lynch, Robert

Worked for more than 160 Hours: Yes

Contribution to Project:

Name: Lee, Christopher

Worked for more than 160 Hours: Yes

Contribution to Project:

Name: Meyrand, Romain

Worked for more than 160 Hours: Yes

Contribution to Project:

Undergraduate Student

Name: Sinenian-Gerdabad, Nareg

Worked for more than 160 Hours: Yes

Contribution to Project:

Name: James, Alexander

Worked for more than 160 Hours: Yes

Contribution to Project:

Name: Vu, Aimee

Worked for more than 160 Hours: No

Contribution to Project:

Name: Gosselin, Jordan

Worked for more than 160 Hours: $\quad$ Yes

Contribution to Project:

Name: Snipes, Erica

Worked for more than 160 Hours: Yes

Contribution to Project:

Name: McClenaghan, Joseph

Worked for more than 160 Hours: Yes 


\title{
Contribution to Project:
}

Technician, Programmer

Name: Kabantsev, Andrey

Worked for more than 160 Hours: Yes

Contribution to Project:

Research physicist

Name: Anderegg, Francois

Worked for more than 160 Hours: Yes

Contribution to Project:

Research physicist

\section{Other Participant}

Name: Christina, Jo Ann

Worked for more than 160 Hours: Yes

Contribution to Project:

\section{Research Experience for Undergraduates}

\author{
Dr. John Bollinger - NIST/Boulder \\ Dr. M.J. Jensen - NIST/Boulder \\ Dr. T. Hasegawa - NIST/Boulder \\ Prof. Igor Kotelnikov - Budker Institute of Nuclear Physics, Russia \\ Dr. Tihiro Ohkawa - Adjunct Professor, UCSD \\ Dr. Yuri Tsidulko - Budker Institute of Nuclear Physics, Russia \\ Dr. Befros Afeyan - Polymath Research Inc.
}

\section{Organizational Partners}

\section{Other Collaborators or Contacts}

\section{Activities and Findings}

\section{Research and Education Activities:}

This research focuses on fundamental processes in plasmas, and emphasizes problems for which precise experimental tests of theory can be obtained. The research program represents a long-term theoretical and experimental collaboration between 3 senior faculty. Experiments are performed on non-neutral plasmas, utilizing three electron traps and one ion trap with a broad range of operating regimes and diagnostics. Theory is focused on fundamental plasma and fluid processes underlying collisional transport and fluid turbulence, using both analytic techniques and medium-scale numerical simulations.

The simplicity of these systems allows a depth of understanding and a precision of comparison between theory and experiment which is rarely possible for neutral plasmas in complex geometry. Moreover, the strong interaction between theory and experiment provides an exceptionally rich environment for training students and post-docs. This collaboration between theory and experiment also extends to the atomic physics experiments at NIST Boulder, the dusty plasma experiments at U. Iowa, and to anti-matter experiments at CERN, through collaborative projects and through exchange of postgraduate researchers.

\section{Findings:}

The recent work has focused on three areas in basic plasma physics. First, experiments and theory have probed fundamental characteristics of 
plasma waves: from the low-amplitude thermal regime, to inviscid damping and fluid echoes, to cold fluid waves in cryogenic ion plasmas. Second, the wide-ranging effects of dissipative separatrices have been studied experimentally and theoretically, finding novel wave damping and coupling effects and important plasma transport effects. Finally, correlated systems have been investigated experimentally and theoretically: UCSD experients have now measured the Salpeter correlation enhancement, and theory work has characterized the 'guiding center atoms'of antihydrogen created at CERN.

\section{NOVEL WAVES}

Recent theory and simulation has verified the long-standing theory conjecture that Electron Acoustic Waves can persist at large amplitude despite their strong damping at small amplitudes (Valentini, Phys Plasmas 2006). The EAW is a nonlinear wave with a carefully tailored trapped-particle population, and the excitation process must create this population.

This theory has now been applied to the parameter regimes of pure electron and pure ion plasmas, obtaining dispersion curves for EAW modes with various radial mode numbers. The EAW modes are found to interleave the traditional electron plasma mode frequencies, depending on plasma temperature (Valentini, Comm. Nonlin. Sci., 2008).

This theory work has led to experiments on both electron plasmas and ion plasmas, which have verified the phase velocity and decay-instabilities predicted for the EAW modes. Interestingly, the experiments also demonstrate that this same decay instability is generic to traditional electron plasma modes as well; experiments are underway to characterize this decay.

Recent experiments on laser-diagnosed pure ion plasmas have provided the first experimental measurements of the ion distribution function coherent with the EAW wave, directly imaging phase space. The measurements quantify the unusual relation between fluid velocities and particle velocities expected in EAWs, and exhibit the crucial trapping plateaux around the phase velocity (Anderegg NNP09, PRL09, Phys Plasmas 09).

Moreover, the experiments are finding unanticipated mode frequency shifts in high temperature regimes. The EAW modes are inherently less frequency-determinate than Langmuir waves, due to the unusual 'negative dynamical compressibility' of the mode. The mode pressure almost exactly cancels the mode electric field, making the mode frequency very sensitive to the particle velocity distribution function. These results may be of direct interest to studies of laser-plasma interactions.

Waves in nonneutral plasmas occur even in the strongly collisional, or fluid limit; but the connection between collisionless theory and fluid theory has been obscure. In recent work (Anderson, Phys Plasmas 2007) Dougherty's simplified Fokker-Planck collision operator leads to a complete set of eigenfunctions, and the eigenvalue spectrum can be physically interpreted to give the sound speed, thermal conductivity, and viscosity as typically considered in the fluid regime. This work has applicability to the rotating-wall confinement technique for cold and strongly-correlated plasmas.

This theory work has now been extended to give the dispersion equation for electron plasma waves over the full range of possible collisionality. In the limit of weak collisionality, Landau damping is recovered. In the strongly collisional regime, theory matches onto fluid results, with an enhancement due to cross-field transport resulting from long-range ExB drift collisions (Anderson NNP09).

Experiments on laser-cooled ion plasmas have now made preliminary measurements of this Langmuir mode damping over a temperature range of $10^{\wedge}-5<\mathrm{T}<1 \mathrm{eV}$. This clearly shows linear and non-linear Landau damping at high temperatures, trapped-particle-mediated damping effects at moderate temperatures, and what may be collisional damping for temperatures below $10^{\wedge}-2 \mathrm{eV}$. We hope to make quantitative comparisons to the collisional damping theory described above.

In other wave experiments, we have established that transiting ions can destabilize the nominally stable diocotron modes in pure electron plasmas (Kabantsev, Trans. Fusion 2007). This instability is similar to instability of curvature-driven flute modes in neutral plasmas, and is also of direct relevance to transiting-particle instabilities in positron-pBar plasmas for anti-hydrogen production at CERN.

\section{TRAPPED-PARTICLE-MEDIATED EFFECTS}

Recent experiments have demonstrated that weak magnetic or electric 'barriers' create small populations of axially trapped particles which have inordinately large bulk plasma effects. The trapped and passing particles experience different dynamics in response to naturally occurring theta-asymmetries or to waves, causing discontinuities in velocity-space. Weak collisional scatterings then cause strong particle transport and strong wave damping. Since weak barriers and weak theta-asymmetries occur in all long plasma traps, these effects are ubiquitous and often dominant.

Our experiments have now established that this trapping-separatrix

1) enables and damps the 'Trapped Particle Diocotron Mode'; 
2) damps $\mathrm{m}>0 \mathrm{kz}>0$ plasma modes; and

3) adds a new dissipative term in resonant 3-wave couplings.

When external trapping asymmetries such as magnetic tilt are added, the separatrix

4) damps $m>0 \mathrm{kz}=0$ diocotron modes;

5) damps $m=0 \mathrm{kz}>0$ plasma modes; and

6) causes bulk plasma expansion and loss.

The resonant 3-wave couplings experiment (Kabantsev, PRL 2008) characterizes a nonlinear dissipative wave coupling term which has not yet been described by theory. Here, experiments give quantitative, low-noise measurements of wave couplings quantifying the new term, and we believe significant new theory will be motivated by these results.

In a theory collaboration motivated by these experiments, Kotelnikov had analyzed the plasma density and potential perturbations arising from a weak axisymmetric magnetic perturbation (Kotelnikov, Phys. Plas. 2006). This work is now being extended to include non-axisymmetric separatrices, motivated by our experimental results.

The three different theoretical approaches of Kotelnikov, Tsidulko and O'Neil are now coming together to provide experimentally testable insights into wave damping by separatrix dissipation. One key theoretical prediction, the near-discontinuous perturbation of the distribution function at the separatrix velocity, may well be testable on our laser-diagnosed ion plasmas.

In a separate major theory analysis, Dubin has revisited neoclassical transport with the addition of separate trapped-particle populations created by an axisymmetric squeeze potential (Dubin Phys Plasmas 08, NNP09). The separate banana, plateau and fluid regimes are identified, and compared to simulations. Here, the analysis treats collisionality varying over 7 decades and includes the important effects of plasma rotation. This theory perspective relates the NNP transport experiments to the analogous transport theory in stellarators. Comparison to our experiments suggests that important shielding or nonlinearity terms are still missing.

Moreover, several different theory approaches are now being developed to understand the effects of theta-variations on separatrices. In this case, dissipative damping and transport effects occur due to 'chaotic separatrix crossings' and do not depend on collisionality. Given the combination of theory, simulations and experiments, we believe consistency will ensue. Moreover, it appears that this novel form of neoclassical transport may have significant implications for toroidal fusion devices.

In related work, Dubin published a review article on collisional transport processes due to collisions with impact parameters larger than the cyclotron radius ('long-range collisions'). This article summarizes previous theory and experiments, and discusses several open questions, such as the effect of Dawson-Okuda vortices on transport in our plasmas. The work was presented at the 2008 Les Houches Summer School on Long Range Coulomb Interactions.

\section{ANTI-MATTER AND CORRELATIONS}

Recent theoretical work on 'guiding center drift atoms' has discovered that these neutral atoms can be trapped due to their electric polarizability.

Surprisingly, the same electric fields which trap the charged components (such as positrons) also polarize the guiding center anti-hydrogen, and gradients in the field then exert a force on the anti-hydrogen. This trapping can be effective both radially and axially, so this work may have important implications for the ATHENA and ATRAP anti-hydrogen collaborations.

Electromagnetic radiation from these weakly-bound guiding center atoms is minimal and energy loss towards the ground state is accomplished primarily through collisions between the atom and free positrons (Bass, Phys. Plas. 09). With Fokker-Planck theory and Monte-Carlo simulation, we have calculated these collisional energy losses, in both the drift regime and the chaotic motion regime. We find that the fraction of magnetic-field seekers is considerably larger than suggested by estimates of the magnetic moment based on guiding center dynamics alone, due to effects associated with chaotic dynamics. These results have important implications for ongoing anti-matter experiments.

The Salpeter correlation enhancement of collisions predicted for nuclear reactions in stars has now been shown theoretically to apply to strongly-correlated non-neutral plasmas, and has been most recently tested experimentally in our pure ion plasmas. Dubin established theoretically that particle dynamics in strong magnetic fields is directly analogous to collisions in strongly correlated non-neutral plasmas (Dubin, PRL 2005). In strong magnetic fields, cyclotron energy, like nuclear energy, is released only through rare close collisions between charges; and the probability of such collisions is enhanced by plasma screening effects, just as in nuclear reactions. Plasma simulations show enhancements of up to $10^{\wedge} 10$ in cyclotron energy equipartition.

Recent experiments on laser-cooled and laser-diagnosed pure ion plasmas have now measured the perpendicular-to-parallel collision rate, 
spanning from the uncorrelated to the correlated regime. In moderately correlated regimes, we measure collision rates consistent with the Salpeter enhancement which varies exponentially with the correlation factor. In more strongly correlated regimes, even larger enhancements are observed, complicated by radial temperature variations and measurement uncertainties. Future laser upgrades will hopefully enable definitive comparisons well into the strongly coupled regime.

\section{Training and Development:}

The strong interaction between experiment, theory, and simulation in this research program provides an exceptionally rich environment for training students and post-docs. Typically six graduate students, two post-doctoral researchers, and two undergraduate students are financially supported by the NSF grant. Also, we typically have undergraduate physics majors doing supplemental projects in the laboratories, REU students every summer, and visiting foreign students on 2-3 baccalaureate enrichment programs.

\section{Outreach Activities:}

Many students from small colleges have gained research experience in plasma experiments and simulation through participation in the NSF-funded REU program at UCSD. In a summer 2008 REU, Erica Snipes will experience theory and experiments in our group.

Aspects of this plasma research have been integrated into innovative undergraduate classes at UCSD. Dan Dubin developed a new mathematical physics course and textbook based on Mathematica software. Fred Driscoll teaches laboratory electronics and an independent-projects-based course on embedded microprocessors; here, lab tours and laboratory connections significantly enhance the courses.

\section{$\underline{\text { Journal Publications }}$}

A.A. Kabantsev and C.F. Driscoll, "Fast Measurement of Pico-Amp Plasma Flows Using Trapped Electron Clouds", Review of Scientific Instruments, p. 3628, vol. 75, (2004). Published,

S.G. Kuzmin and T.M. O'Neil, "Motion of guiding center drift atoms in the electric and magnetic field of a Penning trap", Physics of Plasmas, p. 012101, vol. 12, (2005). Published,

J.H. Yu, T.M. O'Neil and C.F. Driscoll, "Fluid Echoes in a Pure Electron Plasma", Physical Review Letters, p. 025005, vol. 94, (2005). Published,

Daniel H.E. Dubin, "Magnetically bound states of electronic and positronic hydrogen ions:

The case of zero orbital angular momentum", Physical Review A, p. 022504, vol. 71, (2005). Published,

Daniel H.E. Dubin, "Measurement of Screening Enhancement to Nuclear Reaction Rates Using a

Strongly-Magnetized and Strongly-Correlated Non-neutral Plasma", Physical Review Letters, p. 025002, vol. 94, (2005). Published,

A.A. Kabantsev and C.F. Driscoll, "Confinement Asymmetry and Trapped Particle Effects on Radial Transport in Charged Plasmas", Trans. of Fusion Science and Technology, p. 263, vol. 47, (2005). Published,

Daniel H.E. Dubin, "Displacement eigenmodes of cold-fluid and warm-fluid magnetized plasma oscillations", Physics of Plasmas, p. 042107, vol. 12, (2005). Published,

M.J. Jensen, T. Hasegawa, J.J. Bollinger and D.H.E. Dubin, "Rapid Heating of a strongly Coupled Plasma Near the Solid-Liquid Plasma Transition", Physical Review Letters, p. 025001, vol. 94, (2005). Published,

J.H. Yu, C.F. Driscoll, and T.M. O'Neil

, "Phase Mixing and Echoes in a Pure Electron Plasma", Physics of Plasmas, p. 055701, vol. 12, (2005). Published,

N. Shiga, F. Anderegg, D.H.E. Dubin, C.F. Driscoll, and R.W. Gould, "Thermally Excited Fluctuations as a Pure Electron Plasma Temperature Diagnostic", Physics of Plasmas, p. 022109, vol. 13, (2006). Published,

F. Valentini, T.M. O'Neil and D.H.E. Dubin, "Excitation of Nonlinear Electron Acoustic Waves", Physics of Plasmas, p. 052303, vol. 13, (2006). Published, 
A.A. Kabantsev and C.F. Driscoll, "Trapped-Particle-Mediated Collisional Damping of Non-Axisymmetric Plasma Waves", Physical Review Letters, p. 095001, vol. 97, (2006). Published,

F. Valentini, T.M. O'Neil, and D.H.E. Dubin, "Excitation and Decay of Electron Acoustic Waves", American Institute of Physics Conference Proceedings: Non-Neutral Plasma Physics VI, p. 3, vol. 862, (2006). Published,

A. A. Kabantsev and C.F. Driscoll, "Trapped-Particle-Mediated Collisional Damping of Non-axisymmetric

Plasma Waves", American Institute of Physics Conference Proceedings: Non-Neutral Plasma Physics VI, p. 89, vol. 862, (2006). Published,

E.M. Bass and D.H.E. Dubin, "Relaxation of Antihydrogen from Rydberg to Ground State", American Institute of Physics Conference Proceedings: Non-neutral Plasma Physics VI, p. 147, vol. 862, (2006). Published,

A.A. Kabantsev, F. Valentini and C.F. Driscoll, "Experimental Investigation of Electron-acoustic Waves in Electron Plasmas", American Institute of Physics Conference Proceedings: Non-Neutral Plasma Physics VI, p. 13, vol. 862, (2006). Published,

M.W. Anderson and T.M. O'Neil, "Eigenfunctions and Eigenvalues of the Dougherty Collision Operator", Physics of Plasmas, p. 052103 , vol. 14, (2007). Published,

I.A. Kotelnikov, M. Rome, and A. Kabantsev, "Non-neutral plasma equilibria with weak axisymmetric magnetic perturbations", Physics of Plasmas, p. 092108, vol. 13, (2006). Published,

A.A. Kabantsev and C.F. Driscoll, "Ion-Induced Instability of Diocotron Modes in Electron Plasmas Modelling Curvature-Driven Flute Modes", Trans. of Fusion Science and Technology (American Nuclear Society), p. 96, vol. 51, (2007). Published,

I. Kotelnikov, M. Rome and A.A. Kabantsev, "Equilibrium of Charged Plasmas with Weak Axisymmetric Magnetic Perturbations", Trans. of Fusion Science and Technology (American Nuclear Society), p. 238, vol. 51, (2007). Published,

M.W. Anderson and T.M. O'Neil, "Collisional Damping of Plasma Waves on a Pure Electron Plasma Column", Physics of Plasmas, p. 112110 , vol. 14, (2007). Published,

A.A. Kabantsev, T.M. O'Neil, Yu.A. Tsidulko, and C.F. Driscoll, "Resonant Drift-Wave Coupling Modified by Nonlinear Separatrix Dissipation", Physical Review Letters, p. 065002, vol. 101, (2008). Published,

D.H.E. Dubin, "Modeling Nuclear Fusion in Dense Plasmas Using a Cryogenic Non-Neutral Plasma", Physics of Plasmas, p. 055705, vol. 15, (2008). Published,

D.H.E. Dubin, "Theory and Simulations of Electrostatic Field Error Transport", Physics of Plasmas, p. , vol. , (2008). Accepted,

F. Valentini, T.M. O'Neil and D.H.E. Dubin, "Decay Instability of Electron Acoustic Waves", Communications in Nonlinear Science and Numerical Simulation, p. 215, vol. 13, (2008). Published,

D.H.E. Dubin, "Theory and Simulations of Electrostatic Field Error Transport", Physics of Plasmas, p. 07211, vol. 15, (2008). Published,

F. Anderegg, C.F. Driscoll, D.H.E. Dubin and T.M. O'Neil, "Electron Acoustic Waves in Pure Ion Plasmas", Non-Neutral Plasma Physics VII, AIP Conf. Proc., p. 89, vol. 114, (2009). Published,

M.W. Anderson and T.M. O'Neil, "Collisional Damping of Plasma Waves on a Pure Electron Plasma", Non-Neutral Plasma Physics VII, AIP Conf. Proc., p. 114, vol. 114, (2009). Published,

Daniel H.E. Dubin, "Theory and Simulation of Neoclassical Transport Processes with Local Trapping", Non-Neutral Plasma Physics VII, AIP Conf. Proc., p. 121, vol. 114, (2009). Published, 
E.M. Bass and D.H.E. Dubin, "Antihydrogen Formation from Antiprotons in a Pure Positron Plasma", Physics of Plasmas, p. 012101, vol. 16, (2009). Published,

F. Anderegg, D.H.E. Dubin, T.M. O'Neil, and C.F. Driscoll, "Measurement of Correlation-Enhanced Collision Rates", Physical Review Letters, p. 185001, vol. 102, (2009). Published,

F. Anderegg, C.F. Driscoll, D.H.E. Dubin, and T.M. O'Neil, "Wave-Particle Interactions in Electron Acoustic Waves in Pure Ion Plasmas", Physical Review Letters, p. , vol. 095001, (2009). Published,

F. Anderegg, C.F. Driscoll, D.H.E. Dubin, T.M. O'Neil, and F. Valentini, "Electron Acoustic Waves in Pure Ion Plasmas", Physics of Plasmas, p. 055705, vol. 16, (2009). Published,

D.H.E. Dubin, "Plasma Collisional Transport", Long-Range Interacting Systems: Lecture Notes of the Les Houches Summer School (T. Dauxois, S. Ruffo and T. Cugliando, editors), p. 381, vol. 90, (2009). Accepted,

\section{Books or Other One-time Publications}

\section{Web/Internet Site}

\section{URL(s):}

http://nnp.ucsd.edu

\section{Description:}

This site describes the research with both technical and lay-oriented articles, and striking pictures and movies. The site has attracted a wide range of readers, and initiated many productive scientific contacts.

\section{Other Specific Products}

\section{Product Type:}

\section{Instruments or equipment developed Product Description:}

The "rotating wall" confinement technique developed at UCSD is now finding application in labs world-wide, in the areas of plasma physics, atomic physics, and antimatter production. For this technique, weak voltages are applied to the walls of the trap, and these voltages are made to rotate with time. The result is that the rotating non-neutral plasma tends to rotate with the wall voltages, rather than slowing down and thereby being lost. With this technique, single species plasmas can be confined essentially "forever," limited only by chemistry with neutral atom contaminants in the trap.

\section{Sharing Information:}

The ion trapping and time standards group at NIST Boulder incorporated the rotating wall technique immediately after it was developed, containing crystallized ions for arbitrarily long times. The research group of Cliff Surko at UCSD is continuing rotating wall development, with application towards long-time storage of positrons at high densities. This positron application is also being pursued at FirstPoint Scientific, Inc. Professor Robert Pollock at the Indiana University Cyclotron Facility utilized the rotating wall to maintain a plasma target for high-energy beams. The ATHENA group at CERN utilizes the rotating wall technique in a nested trap, producing antihydrogen by the recombinatin of positrons. The HiPAT antiproton storage group at Marshall Space Flight Center was advised by the UCSD group to incorporate a rotating wall in their next apparatus modification, and they have applied for a patent on it.

\section{Contributions}

\section{Contributions within Discipline:}

The camera-diagnosed apparatus 'Cam-V' opened the field of quantitatively-imaged 2D fluid turbulence using electron plasmas; the apparatus is now proving similarly effective in quantitative measurements of trapped-particle effects and asymmetry-induced transport. The laser-diagnosed ion apparatus 'IV' established a new standard for quantitative measurement of plasma transport properties, and is now exploring correlation effects on collisions at milli-Kelvin plasma temperatures. The 'rotating wall' confinement technique has revolutionized 
non-neutral plasma experiments, enabling infinite-time containment, and has been adopted at essentially all other laboratories doing similar work.

The theory work applies to a broad range of neutral and non-neutral plasmas and is finding direct application in anti-matter production and confinement. UCSD theory work has characterized the energy level and dynamics of 'guiding-center drift atoms' which represent a significant barrier on the path to the desired ground-state antihydrogen. Theory and simulations now extend into the more tightly bound chaotic regime where relaxation to the ground state becomes rapid. Moreover, the theory offers the exciting possibility of 'polarization trapping' of the neutral atoms, and has also suggested 'minimum-B' traps which do not have the problematical large-scale magnetic field asymmetries of standard designs.

A major focus of theory, simulations and experiments has been on wave damping and particle transport effects in plasmas where small magnetic or electrostatic ripples cause localized particle trapping. Experimentally, Trapped-Particle-Mediated effects have been quantitatively measured for damping of most plasma modes, and the bulk plasma experiment loss from overall trap asymmetries has been measured. Using theory supported by simulations, Dubin has developed a broad overview of neo-classical transport, and this theory is now being quantitatively compared to experiments. The comparison necessarily involves understanding the nonlinear shielding response of the plasma. This promises to be a uniquely complete description of plasma transport relevant to neutral plasmas also.

\section{Contributions to Other Disciplines:}

Research with these plasmas has proved to be remarkably interdiscipinary, contributing to and borrowing from atomic physics, condensed matter physics, and fluid dynamics.

The rotating wall technique has enabled new classes of precision spectroscopy experiments in the atomic physics group at NIST Boulder. This technique is now routinely used for the compression of positrons in the antihydrogen efforts at CERN, and is being developed by Surko and collaborators for storage of large numbers of positrons in honeycomb traps.

The new theory of polarization trapping of guiding center atoms of anti-hydrogen offers exciting experimental possibilities, and we anticipate close interaction between theory and experiment in the atomic physics and plasma physics communities. Similarly, the new theory of 'guiding center ions' offers the possibility of novel atomic states in high magnetic field traps. Here, we expect that new manipulation and diagnostic tehniques will be developed as knowledge of these states increases. Already, theory suggests that detection of these ions would imply that the underlying atom is deeply bound.

The new UCSD research program on trapped particle modes and asymmetry-induced transport has wide-ranging applicability in single-species plasmas, shows direct relevance to the substantially more difficult problems of asymmetry-induced (bootstrap) currents and transport in fusion plasmas, and may offer insight into difficult problems in space plasmas. Localized trapping occurs in virtually all plasmas, and simple paradigms for trapped-particle-mediated effects may have wide applicability.

The theory analysis of normal modes in ellipsoidal plasmas has proved directly applicable to the cryogenic trapped ion plasmas used extensively in atomic physics. The interdisciplinary nature of this research was highlighted by the 2000 APS Excellence in Plasma Physics Award to Dan Dubin and John Bollinger (an atomic physicist) for theory and application of these modes to ion clusters. This collaboration may continue with attempts to detect the novel modes recently theorized.

The theory work on 3D Coulomb crystals connects intimately with the one-component plasma, which is an important paradigm of condensed matter physics. Most recently, the energy equipartition rate in strongly correlated non-neutral plasmas has been theoretically connected to the nuclear reaction rate in dense neutral plasmas, and collisional enhancements of up to 10 billion have been predicted. Preliminary experiments on the IV apparatus provide the first experimental verification of this effect, and may well help decide between competing theories. This work has substantial cross-disciplinary connections with nuclear physics and astrophysics.

The 2D vortices and waves in electron plasmas are directly analogous to 2D vortices and waves in incompressible inviscid fluids, and connections to the fluids community are strong and continuing. Research connections to atmospheric physics groups remain strong.

\section{Contributions to Human Resource Development:}

The strong interaction between theory and experiment in this research program provides an exceptionally rich environment for training students and post-docs. Approximately six graduate students, two post-doctoral researchers, and two undergraduate students are supported by the NSF grant in any given year.

After their Ph.D. research, recent graduate students have begun careers in fields ranging over plasma physics, atmospheric physics, atomic 
physics, and biophysics. Undergraduate students gain significantly from exposure to the well-diagnosed computer-based plasma experiments in our laboratories, and from contributing to the medium scale computation and simulations used by the theorists.

Tours of the IV laser and CamV labs continue to be popular for physics majors in several UCSD classes, hopefully conveying the excitement of advanced physics research to students considering graduate school. The one- and two-quarter independent study courses and summer REU programs allow students to experience research directly.

\section{Contributions to Resources for Research and Education:}

The UCSD group has freely shared resource information and technologies for a wide range of charged-particle traps in research and education groups worldwide.

\section{Contributions Beyond Science and Engineering:}

Theory and experimental members of the research group routinely contribute advice to the lay public, to plasma industry and to legal practitioners on a variety of topics ranging from automobile and airplane crashes, to technologies for plasma processing of waste.

\section{Conference Proceedings}

Organizational Partners

Categories for which nothing is reported:

Any Book

Any Conference 